\title{
Loss of splicing factor IK impairs normal skeletal muscle development
}

\author{
Hye In Ka ${ }^{1,2+}$, Hyemin $\mathrm{Seo}^{3 \dagger}$, Youngsook Choi ${ }^{2}$, Joohee Kim ${ }^{1}$, Mina Cho ${ }^{1}$, Seok-Yong Choi ${ }^{4}$, Sujeong Park', \\ Sora Han ${ }^{2}$, Jinsu An ${ }^{5,6}$, Hak Suk Chung ${ }^{5,6}$, Young Yang ${ }^{1,2^{*}}$ (i) and Min Jung Kim ${ }^{\text {* }^{*}}$
}

\begin{abstract}
Background: IK is a splicing factor that promotes spliceosome activation and contributes to pre-mRNA splicing. Although the molecular mechanism of IK has been previously reported in vitro, the physiological role of IK has not been fully understood in any animal model. Here, we generate an ik knock-out (KO) zebrafish using the CRISPR/ Cas9 system to investigate the physiological roles of IK in vivo.

Results: The ik KO embryos display severe pleiotropic phenotypes, implying an essential role of IK in embryonic development in vertebrates. RNA-seq analysis reveals downregulation of genes involved in skeletal muscle differentiation in ik KO embryos, and there exist genes having improper pre-mRNA splicing among downregulated genes. The ik KO embryos display impaired neuromuscular junction (NMJ) and fast-twitch muscle development. Depletion of ik reduces myod 1 expression and upregulates pax7a, preventing normal fast muscle development in a non-cell-autonomous manner. Moreover, when differentiation is induced in IK-depleted C2C12 myoblasts, myoblasts show a reduced ability to form myotubes. However, inhibition of IK does not influence either muscle cell proliferation or apoptosis in zebrafish and C2C12 cells.
\end{abstract}

Conclusion: This study provides that the splicing factor IK contributes to normal skeletal muscle development in vivo and myogenic differentiation in vitro.

Keywords: IK, Zebrafish, CRISPR/Cas9, Skeletal muscles, Myogenesis

\section{Background}

Precursor messenger RNA (pre-mRNA) splicing, which occurs in most eukaryotes, is a process to remove noncoding regions (introns) and connect the remaining coding regions (exons) in the nucleus. Pre-mRNA splicing is performed by a sequential assembly of five small nuclear RNA-proteins (snRNPs) called the spliceosome [1-4]. Briefly, the U1 and U2 snRNPs recognize the $5^{\prime}$ splice site (SS) and branch site (BS) of introns, respectively, and form the A complex. Next, U4/5/6 tri-snRNP binds to it to form the pre-catalytic B complex. After

\footnotetext{
*Correspondence: yyang@sookmyung.ac.kr; minkim@sookmyung.ac.kr

${ }^{\dagger}$ Hye In Ka and Hyemin Seo contributed equally to this work.

'Department of Biological Sciences, Sookmyung Women's University, Seoul 04310, Republic of Korea

Full list of author information is available at the end of the article
}

activation of the B complex by multiple catalytic steps, it is converted to the $\mathrm{B}^{\text {act }}$ complex, which performs the splicing event. For the splicing event, both snRNPs and non-snRNPs are required. As pre-mRNA splicing contributes to protein diversity, disruptions in the splicing mechanism lead to various pathological disorders including retinitis pigmentosa, Hutchinson-Gilford progeria syndrome, amyotrophic lateral sclerosis, muscular dystrophy such as in Duchenne muscular dystrophy and spinal muscular atrophy, and abnormal craniofacial development such as in Burn-McKeown syndrome and Nager syndrome [5-9]. Although a number of splicing factors have been studied in diverse diseases, their precise mechanism is largely unknown, and further studies are required to investigate their function. 
IK, also known as RED protein because of its arginine (R)-, glutamic acid (E)-, and aspartic acid (D)-rich domain, participates in the regulation of cell mitotic kinases and phosphatases [10] and localization of the spindle assembly checkpoint protein MAD1 to the kinetochore [11]. Furthermore, it plays a role in pre-mRNA splicing by interacting with SMU1, a B complex-specific protein. The interaction between IK and SMU1 leads to mutual stabilization, whereby the stabilized complex performs the splicing function as a unit [12]. A previous study has reported that IK and SMU1 are recruited for viral RNA polymerase gene expression for alternative splicing of the viral mRNAs during influenza virus infection [13]. It has been revealed that IK and SMU1 perform precise splicing if the distance of the branch between $5^{\prime}$ SS and BS is dominantly shorter than 200 nts [14]. Although IK has been reported to function as a splicing factor, little is known about the mechanism by which IK functions in vivo and the tissue susceptible in its absence. To elucidate the functional role of IK during development, we established, for the first time, the $i k$ knock-out (KO) zebrafish model with the CRISPR/Cas9 system.

The skeletal muscle is a highly organized tissue and is generated through a process called myogenesis [15]. During embryonic myogenesis, mesoderm-derived myoblasts fuse into multinucleate muscle fibers. These myogenic progenitor cells are quiescent and convert into mature skeletal muscles with the ability to function as muscles [16, 17]. As mature muscles have the capacity to regenerate after damage, it is critical to establish normal muscles in the embryo stage. In myogenesis, the expression of myogenic regulatory factors (MRFs), including MyoD, Myf5, myogenin (MyoG), and Mrf4, regulates myogenic proliferation and differentiation [1820]. The loss of MRF function causes the failure of myogenesis with parallel upregulation of Pax3/7 [21]. When myoblasts begin to generate multinucleated myotubes, MyoG and myosin heavy chain (MHC) induce the terminal differentiation of myoblasts [22]. Thus, the depletion of these genes results in reduced myogenic capacity and abnormal muscle development in vivo [19, 23]. In this study, we propose that loss of splicing factor IK results in abnormal pre-mRNA splicing of genes involved in skeletal muscle differentiation and reduces the ability to form normal skeletal muscle during myogenesis.

\section{Results}

CRISPR/Cas9-mediated ik KO embryos display abnormal phenotypes and lethality

Zebrafish contains a single copy of $i k$ (Genebank Accession number BC049322.1) on chromosome 21; the encoded protein is 548 amino acids in length. Zebrafish IK protein shows $82 \%$ identity and $92 \%$ similarity with the human, mouse, and chicken IK protein (Fig. 1a). Amino acids 353-363 in human IK protein are missing in zebrafish IK protein, whereas all the functional $\mathrm{N}$ terminal RED domains are highly conserved (Additional file 1: Figure S1). To explore the in vivo function of IK during zebrafish development, we applied CRISPR/ Cas 9 technology to generate $i k \mathrm{KO}$ zebrafish by targeting the RED domain. We selected an indel mutation in exon 2 . Sequence analysis revealed that $i k$ mutants carried an 8-bp deletion (ATGAGGTG) and a 10-bp insertion (TCTGGCTCCA) at nucleotide position 53 with premature translational termination, resulting in an abnormally short IK protein (31 amino acids long) (Fig. 1b). As the indel mutation removes a BslI site at 53 nucleotides from the starting codon facilitating easy genotype confirmation for mutant screening, wild type (WT; +/+) alleles were completely digested with BslI enzyme (Fig. $1 c)$. Furthermore, we confirmed $i k$ mRNA was not amplified in homozygous $i k \mathrm{KO}(-/-)$ embryos at 1.5 and 4 days post-fertilization (dpf) using quantitative RT-PCR (qRT-PCR) (Fig. 1d). To determine the effect of $i k \mathrm{KO}$ embryos on development, we observed the morphological changes in the progeny from pairwise crosses of heterozygous zebrafish (Fig. 1e). Until $1 \mathrm{dpf}$ of development, $i k \mathrm{KO}$ embryos appeared indistinguishable from $\mathrm{WT}$ and heterozygous embryos. The $i k \mathrm{KO}$ embryos at $36 \mathrm{~h}$ post-fertilization (hpf) did not swim and began to display severe pleiotropic phenotypes and body deformities including a downward tail curvature, which worsened over time. The $i k \mathrm{KO}$ embryos did not show a significant difference in cardiac structure compared to WT, forming an S-shaped loop as well as a heartbeat until $2 \mathrm{dpf}$ (Additional file 2: Video S1). From $3 \mathrm{dpf}, i k$ $\mathrm{KO}$ embryos started to gradually exhibit weak pericardial edema and a slowed-down heartbeat (24/15 s) compared to that in WT (31/15 s) (Additional file 3: Video S2), and subsequently died at $6 \mathrm{dpf}$. To further confirm that loss of $i k$ did not affect heart structure formation, we injected $i k$ morpholino (MO) into $T g$ (kdrl: GFP) zebrafish embryos which cardiomyocytes express GFP, and observed the cardiac structure at $2 \mathrm{dpf}$ (Additional file 4: Video $\mathrm{S} 3)$. Similar to $i k \mathrm{KO}$ zebrafish embryos, there was no significant difference in cardiac structure between $i k$ MO embryos and WT at the same stage (48 hpf), though the heartbeat rate was slightly slow. These results indicate that IK plays an essential role in zebrafish embryonic development.

\section{RNA-seq analysis of ik $\mathrm{KO}$ embryos reveals downregulation of genes involved in skeletal muscle differentiation}

Next, to investigate whether loss of splicing factor IK affects the transcriptome of $i k \mathrm{KO}$ embryos, we performed RNA-seq analysis to compare the transcriptomes of $i k$ 
A

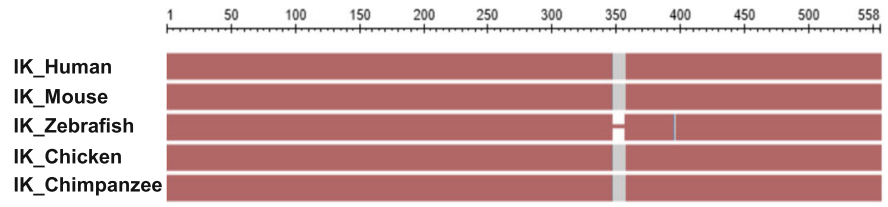

B

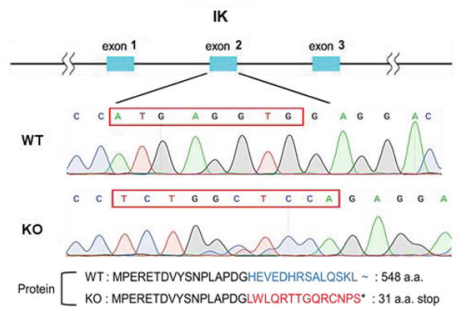

C

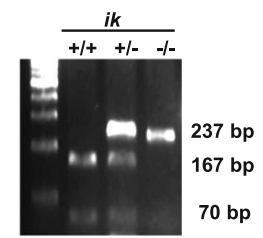

D

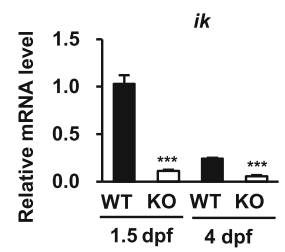

E

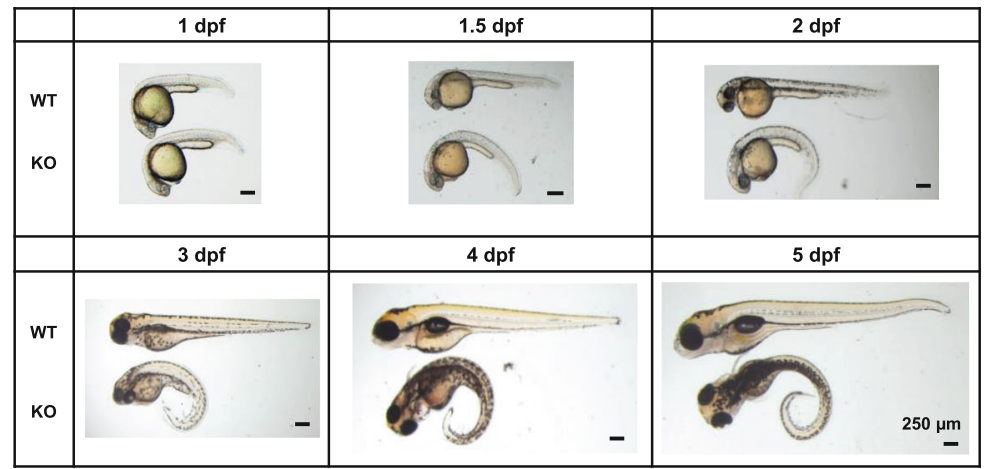

Fig. 1 CRISPR/Cas9-mediated ik knock-out (KO) embryos display abnormal embryonic phenotypes and lethality. a Sequence alignment of IK proteins from human, mouse, zebrafish, chicken, and chimpanzee by NCBI COBALT. The conserved sequence is shown in red. b Schematic of zebrafish ik locus and CRISPR/Cas9 targeted region. The asterisk denotes the stop codon. c Genotype confirmation of ik in wild type (WT), heterozygous, KO embryos by RT-PCR analysis. WT alleles were digested with the Bs/l enzyme. +/+: $70+167 \mathrm{bp},+/-: 70+167+237 \mathrm{bp},-/-: 237$ bp. d Relative mRNA levels of $i k$ in WT and ik KO embryos at 1.5 and 4 days post-fertilization (dpf) using qRT-PCR analysis. The average of three independent experiments is shown with error bars. e Lateral views of WT and $i k$ KO embryos during embryonic development. Scale bar $=250 \mu m$

KO embryos with those of WT embryos at $3 \mathrm{dpf}$. Among the diverse biological process categories, skeletal muscle differentiation was top ranked; $18.18 \%$ of genes related to skeletal muscle differentiation were differentially expressed between WT and $i k \mathrm{KO}$ embryos (Fig. 2a). Additionally, the Spearman correlation coefficient $(R)$ for RNA-seq analysis between WT and $i k \mathrm{KO}$ embryos was 0.95 (Fig. 2b). Among the differentially expressed genes related to skeletal muscle differentiation with adjusted $p$ values of 0.05 and fold change of at least 2 , seven genes are presented in a heat map and analyzed by hierarchical clustering (Fig. 2c, Additional file 5: Table S1). cdkn1a was upregulated and the other 6 genes including mybpc2a, mybpc1, tnnt2e, smyd1a, acta1a, and tnni3k were downregulated. Next, we confirmed the mRNA expression of these genes using qRT-PCR in 3 dpf WT and $i k \mathrm{KO}$ embryos (Fig. 2d). As a result, the relative mRNA expression patterns of these genes were consistent with the RNA-seq results.

\section{ik KO embryos show damaged pre-mRNA splicing events in skeletal muscle differentiation genes}

As IK is a splicing factor implicated in the activation of the B complex [12], we hypothesized that altered mRNA expression in $i k \mathrm{KO}$ embryos may be attributed to improper pre-mRNA splicing. To confirm pre-mRNA splicing aberrations in $i k \mathrm{KO}$ embryos, we determined splicing events by analyzing RNA-seq data and generating Sashimi plots [24]. As a result, 6 skeletal muscle differentiation genes reduced in RNA-seq generally had a lower number of junction reads compared to WT, as visualized by bridges in the Sashimi plots (Additional file 6: Figure S2 A-G). In particular, mybpc2a (Fig. 3a, Additional file 6: Figure S2 B) and mybpc1 (Fig. 3b, 


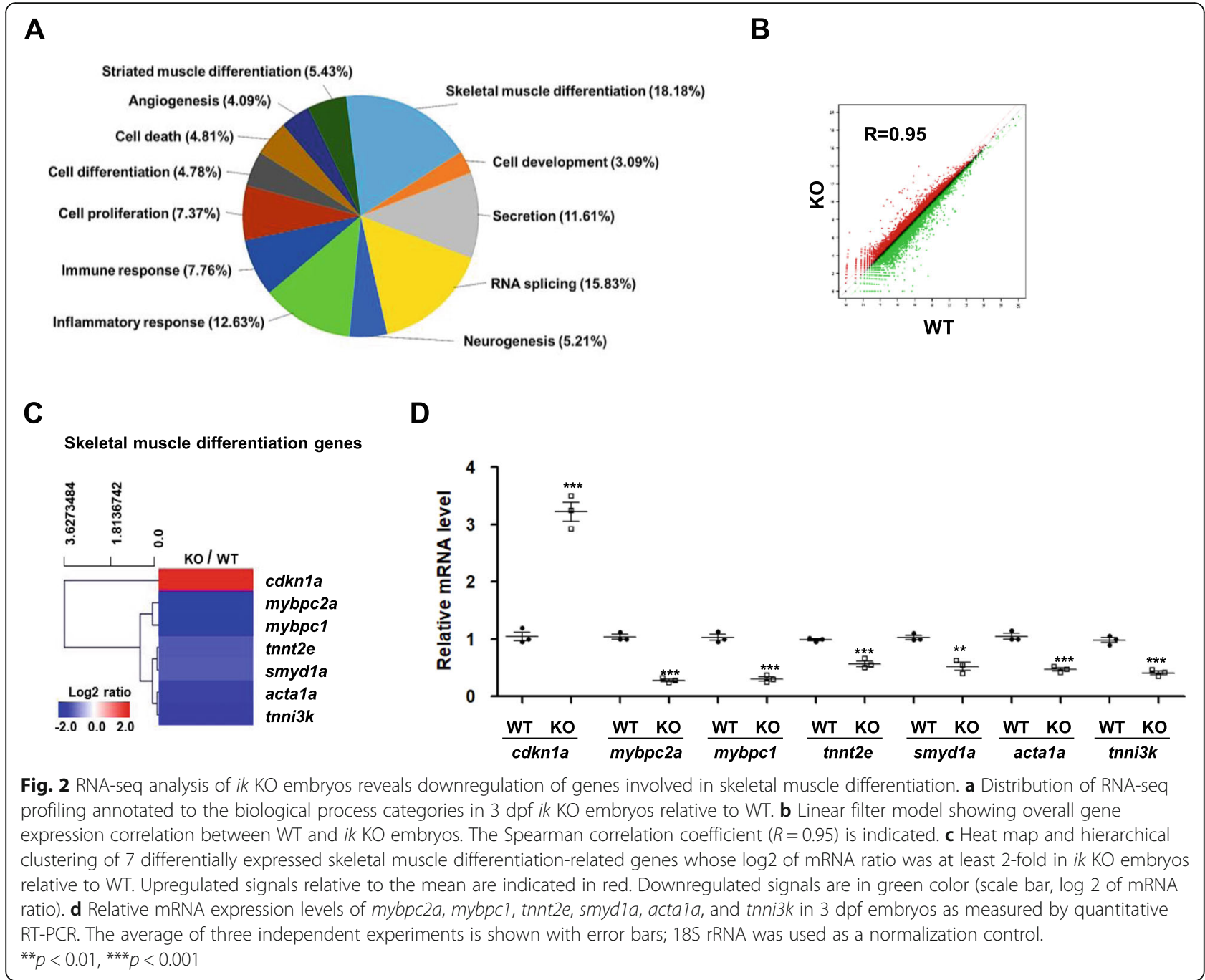

Additional file 6: Figure S2 C) showed several sites wherein unlinked by the bridges. Next, we monitored the splicing events of housekeeping genes as representative examples of universal genes to determine whether the general splicing factor $i k$ also affects splicing of other non-muscle genes. However, there were no splicing defects in housekeeping genes including actb1, gapdh, and tuba1b (Fig. 3c-e, Additional file 7: Figure S3 A-C). Based on Sashimi plot analyses, we examined premRNA phenotypes of skeletal muscle differentiation genes using standard PCR and qRT-PCR with primers flanking the exon regions. As a recent study revealed that IK predominantly splices $<200 \mathrm{nt}$ long introns in vitro [14], we designed primers against exons flanking short introns ( $200 \mathrm{nt})$. As expected, in $i k \mathrm{KO}$ embryos, unspliced pre-mRNA was examined in mybpc2a (E7-E8, E11-E12; Fig. 3f, g), mybpc1 (E7-E8, E15-E16; Fig. 3h, i), and tnnt2e (E6-E7; Fig. 3j, k), which indicated decreased mRNA levels. However, we observed that some short introns such as mybpc1 (E19-E20; Fig. 3h) and tnnt2e (E9-
E10; Fig. 3j) were precisely spliced even though IK was absent. Furthermore, cytoskeleton gene acta1a (E3-E4, E4-E5) splicing was not affected in $i k \mathrm{KO}$ embryos (Fig. $3 \mathrm{l}, \mathrm{m})$, despite the short intron sites. Taken together, $i k$ $\mathrm{KO}$ embryos reveal damaged pre-mRNA splicing events in several genes involved in skeletal muscle differentiation.

Fast-twitch muscle fibers are impaired in ik KO embryos with downregulated myod 1

As splicing events of muscle differentiation-related genes were compromised by $i k$ mutation, we examined whether muscle development was defected in $i k \mathrm{KO}$ embryos. First, through DIC images, we observed that $i k$ $\mathrm{KO}$ embryos had twisted and disorganized muscle fibers (Fig. 4a). Next, transverse sections of the skeletal muscles were prepared and stained with anti-F310 and F59 antibodies to identify fast- and slow-twitch muscle fibers, respectively, in WT and $i k \mathrm{KO}$ embryos. The $i k \mathrm{KO}$ embryos showed significantly lower density and staining 


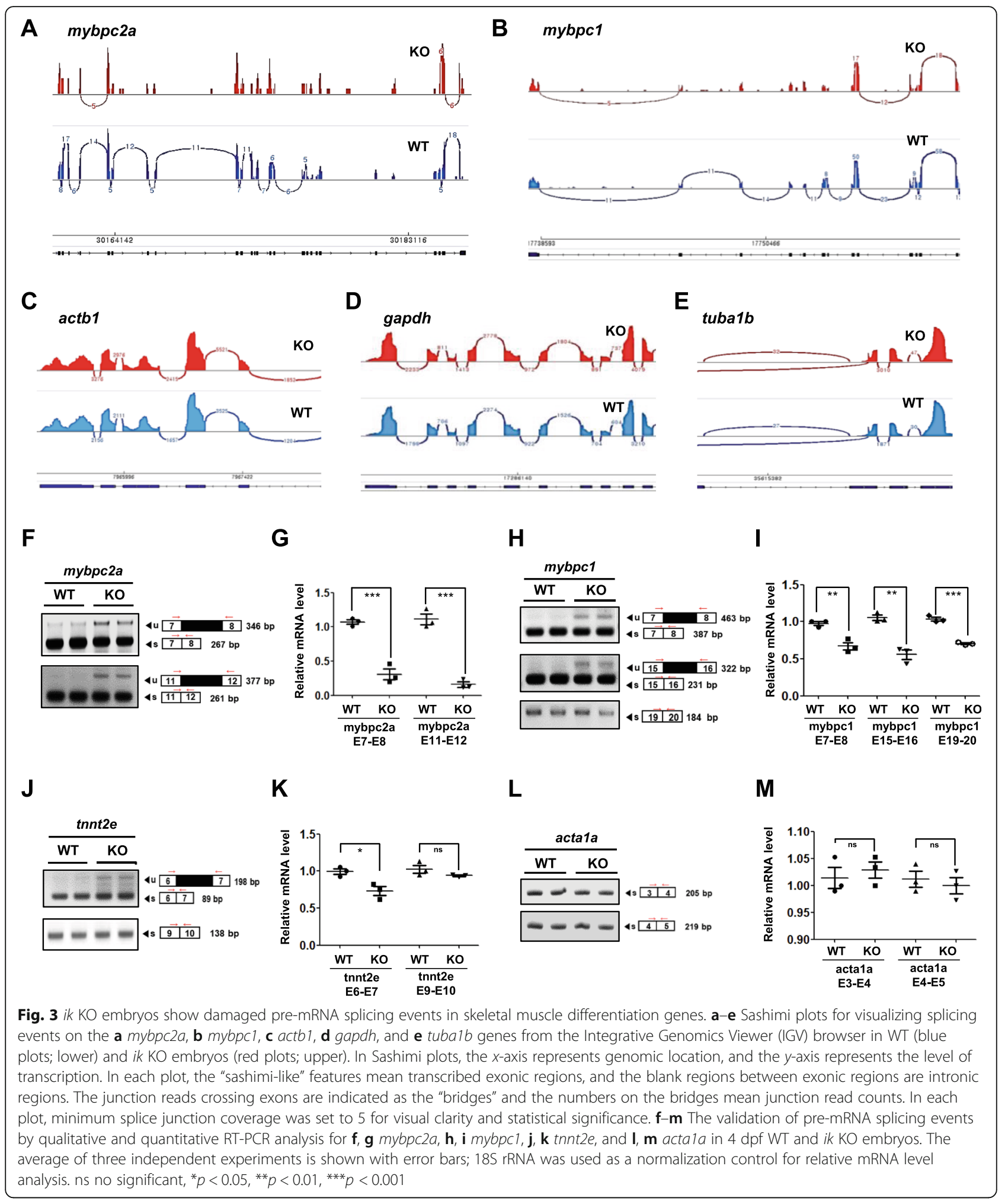

intensity compared to WT, indicating a defect in fasttwitch muscle fibers (F310) (Fig. 4b; left panel). However, no obvious defects were observed in slow-twitch muscle fibers (F59) (Fig. 4b; right panel). Disorganized and defected fast-twitch muscle fibers were observed even in whole-mount immunostained muscle fibers of $i k$ $\mathrm{KO}$ embryos, confirming that IK predominantly affects fast-twitch muscle fiber development in zebrafish (Fig. 


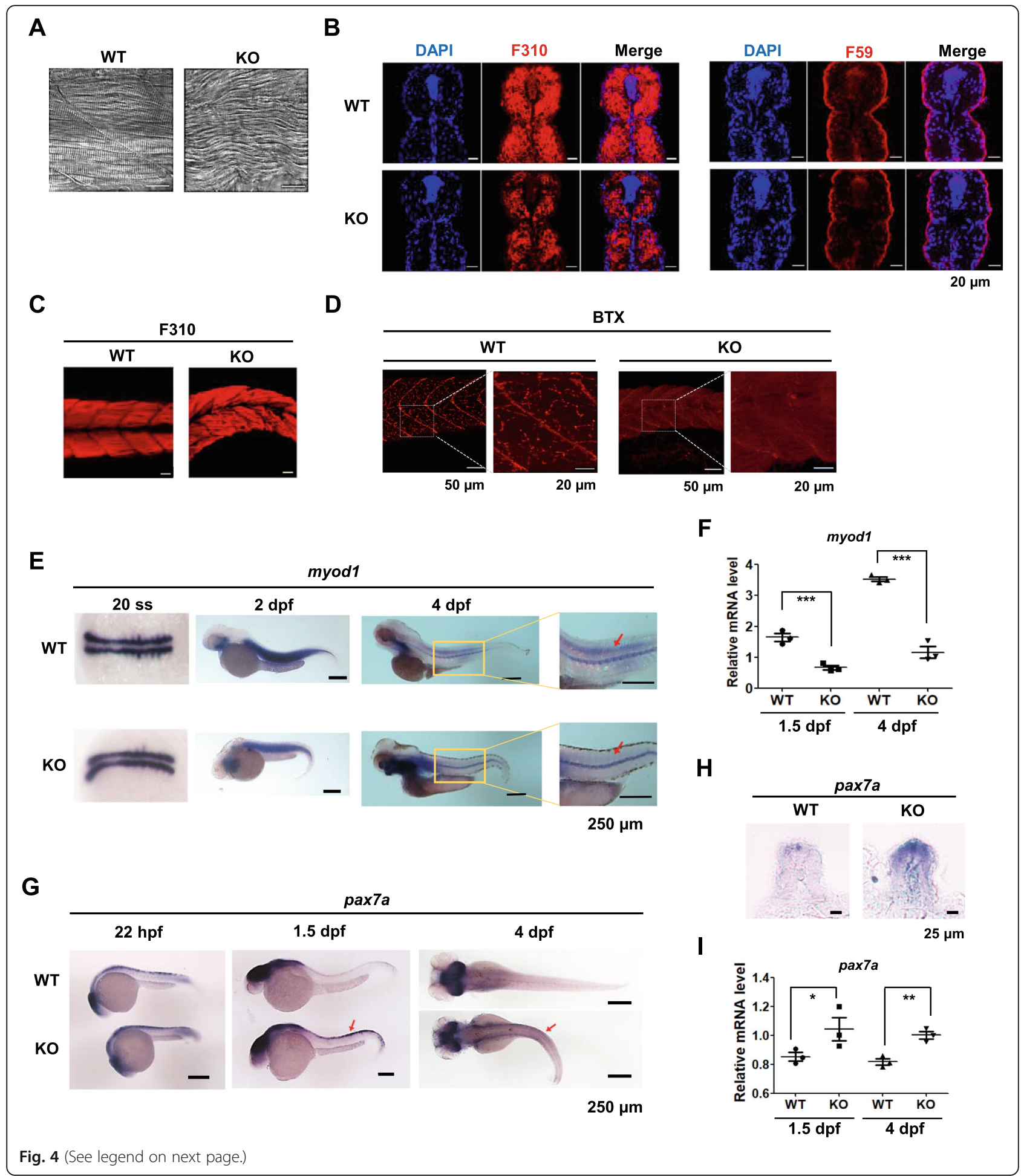


(See figure on previous page.)

Fig. 4 Fast-twitch muscle fibers are impaired in ik KO embryos with downregulated myod1. a DIC confocal images of the skeletal muscle at 3 dpf WT and ik $\mathrm{KO}$ embryos. Scale bar $=20 \mu \mathrm{m}$. b Transverse sections of the skeletal muscle stained with anti-F310 (fast-twitch muscle fibers; left panel) and anti-F59 (slowtwitch muscle fibers; right panel) in $4 \mathrm{dpf}$ WT and $i k \mathrm{KO}$ embryos. Scale bar $=20 \mu \mathrm{m}$. c Whole-mount immunostaining with anti-F310 (fast-twitch muscle fibers) of $4 \mathrm{dpf} \mathrm{WT}$ and $i k \mathrm{KO}$ embryos. Scale bar $=20 \mu \mathrm{m}$. $\mathbf{d}$ Whole-mount immunostaining with a-bungarotoxin (BTX) in $4 \mathrm{dpf}$ WT and $i k$ KO embryos. e Wholemount in situ hybridization for myod1 mRNA in 20 somite stage (ss), and $2 \mathrm{dpf}$ and $4 \mathrm{dpf} \mathrm{WT}$ and $i k \mathrm{KO}$ embryos. The red arrow indicates the position of signal myod1. Scale bar $=250 \mu \mathrm{m}$. $\mathbf{f}$ Relative myod $1 \mathrm{mRNA}$ levels in WT and ik KO embryos at $1.5 \mathrm{dpf}$ and $4 \mathrm{dpf}$. The average of three independent experiments is shown with error bars. *** $<0.001$. g Whole-mount in situ hybridization for pax7a mRNA in WT and ik KO embryos at $22 \mathrm{hpf}, 1.5 \mathrm{dpf}$, and $4 \mathrm{dpf}$. The red arrow indicates the position of signal pax7a. Scale bar $=250 \mathrm{\mu m}$. $\mathbf{h}$ Transverse section of whole-mount in situ hybridization for pax7a in $1.5 \mathrm{dpf}$ WT and ik KO embryos. Scale bar $=25 \mu \mathrm{m}$. i Relative pax7a mRNA levels of 1.5 and $4 \mathrm{dpf}$ WT and ik KO embryos. The average of three independent experiments is shown with error bars. ${ }^{*} p<0.05,{ }^{* *} p<0.01$

4c). Furthermore, defects in the neuromuscular junction (NMJ) were determined by staining with $\alpha$-bungarotoxin (BTX) to label nicotinic acetylcholine receptors. $i k \mathrm{KO}$ embryos had lower staining intensity in NMJ synaptic boutons compared to WT due to the lack of normal muscle development (Fig. 4d). Next, to investigate the molecular mechanisms underlying $i k$-related skeletal muscle development, we analyzed the RNA-seq data for the myogenic regulators in myogenesis. We found that the expression level of the myogenic marker myod 1 was downregulated in the $i k \mathrm{KO}$ embryos in RNA-seq data (Additional file 8: Table S2). Thus, to further confirm whether loss of IK affects muscle development, myod1 expression levels were determined using in situ hybridization and qRT-PCR. In situ hybridization showed that embryonic myod1 expression was reduced in the dorsal domains of the trunk in $i k \mathrm{KO}$ embryos (Fig. 4e). Similarly, qRT-PCR analysis also revealed decreased myod1 mRNA expression in $i k \mathrm{KO}$ embryos (Fig. 4f). In the RNA-seq data, the expression of transcription factor pax $7 a$, which is expressed not only in muscles but also in the nervous system, was increased (Additional file 8: Table S2). Accordingly, we performed in situ hybridization to observe the specific locus of pax $7 a$ in $i k \mathrm{KO}$ embryos, and pax7a was expressed in the dorsal neural tube and the surface of somites. The $i k$ $\mathrm{KO}$ embryos showed a higher staining intensity of pax7a at the superficial layers of somite in early stages, which persisted until $4 \mathrm{dpf}$ (Fig. $4 \mathrm{~g}, \mathrm{~h}$ ). Similarly, the mRNA level of pax7a was increased in $i k \mathrm{KO}$ embryos, as determined by qRT-PCR (Fig. 4i), and there were no changes in pre-mRNA splicing events as deduced by Sashimi plots (Additional file 9: Figure S4). Taken together, abnormal embryonic phenotype in $i k \mathrm{KO}$ embryos was caused by defective skeletal muscle development.

\section{IK is required for normal myogenic differentiation in C2C12 myoblasts}

To address whether IK plays a fundamental role in muscle development in vitro, IK-targeted siRNA was designed and introduced to suppress protein (Fig. 5a) and mRNA (Fig. 5b) levels of IK in undifferentiated $\mathrm{C} 2 \mathrm{C} 12$ myoblast cells. Next, to observe whether depletion of IK might interfere with the $\mathrm{C} 2 \mathrm{C} 12$ myoblast differentiation process, we analyzed the protein levels of MRFs such as MyoD, Pax7, and MyoG in IK-depleted C2C12 cells. Similar to $i k$ KO zebrafish embryos, MyoD was downregulated at the protein level, whereas Pax7 was upregulated (Fig. 5c). Moreover, we could not detect MyoG protein in either control or IK-depleted cells due to undifferentiated status. To confirm whether IK regulates Pax7 and MyoD directly or indirectly, we performed immunoprecipitation where endogenous IK pulled down endogenous Pax7 and MyoD in $\mathrm{C} 2 \mathrm{C} 12$ cell lysates. Endogenous IK directly bound to endogenous Pax7 and MyoD (Additional file 10: Figure S5). Next, to determine whether IK might contribute to normal myoblast differentiation, $\mathrm{C} 2 \mathrm{C} 12$ myoblasts were induced to differentiate for 3 days. When myoblasts halt the proliferation of the myogenic progenitor cells, the myoblasts expressing Myf5 and/or MyoD fuse to form myotubes expressing MyoG and MHC [25]. Thus, after inducing skeletal muscle differentiation, the myoblasts were stained for anti-MHC antibody, a myogenic differentiation marker, to detect myotube formation (Fig. 5d). IKdepleted cells showed about 4-fold lower percent of MHC-positive nuclei per total nuclei in IK-depleted C2C12 cells than control cells, indicating a decreased ability to form myotubes with no change in total nuclei number. To further confirm this result, the expression levels of myogenic specific markers MHC and MyoG were determined during myogenic cell differentiation. Consistent with the immunostaining result, $\mathrm{MHC}$ and MyoG protein levels were also relatively lower in IK-depleted cells than those in control cells on days 2 and 3 after differentiation (Fig. 5e). Furthermore, we measured the MyoD and MyoG mRNA expression levels using qRT-PCR on days 2 and 3 after differentiation. The MyoD and MyoG mRNA expression levels were also reduced (Fig. 5f, g). Collectively, these results indicate that depletion of $\mathrm{IK}$ in $\mathrm{C} 2 \mathrm{C} 12$ myoblasts reduced its ability to form differentiated myotubes.

\section{IK functions in a non-cell-autonomous manner in skeletal muscle cells}

As $i k$ depletion affects the development of muscle, we asked whether cell-autonomous perception of $i k$ is necessary for fast muscle formation. Thus, we performed 


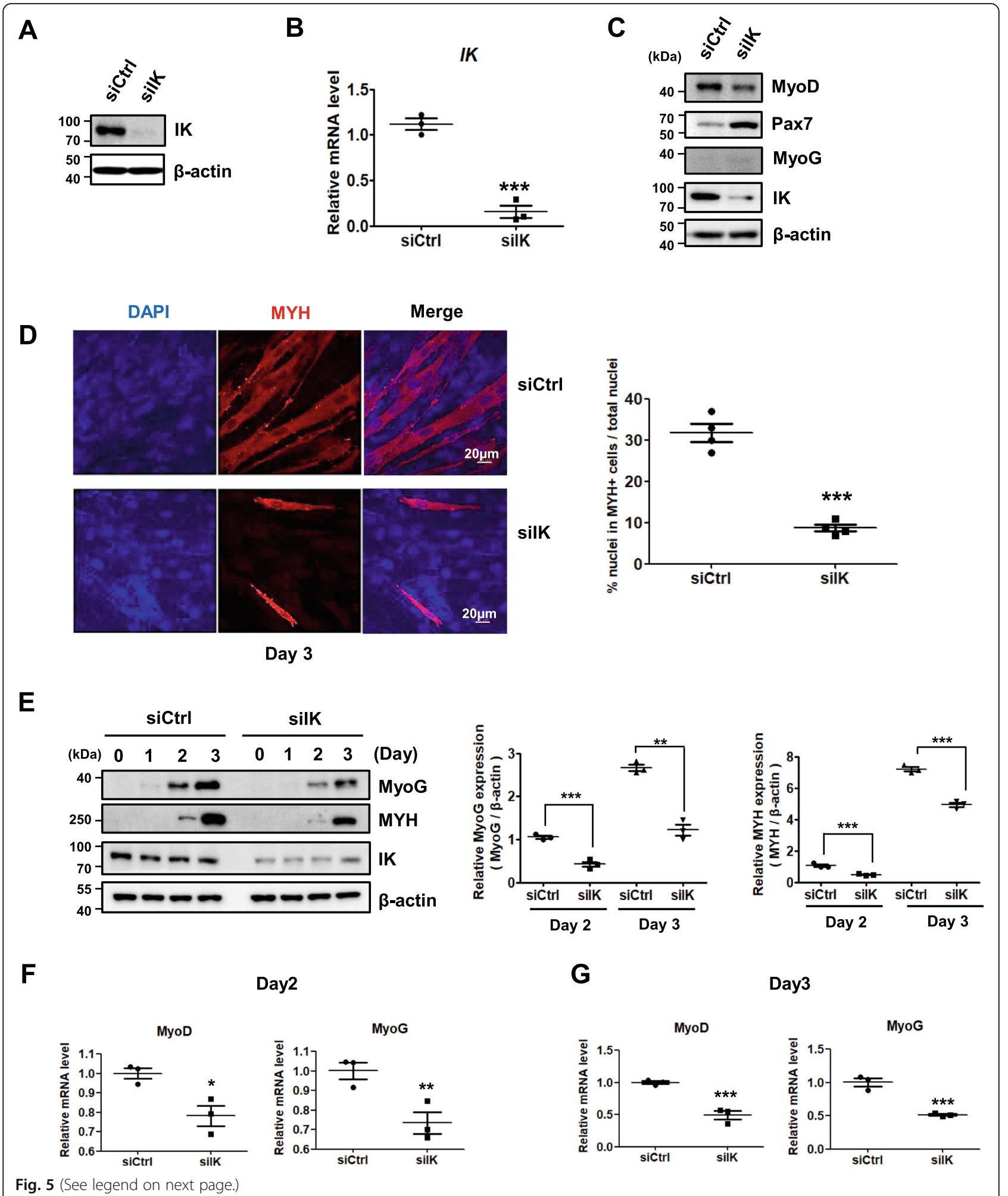


(See figure on previous page.)

Fig. 5 IK-depleted myoblasts have a reduced ability to form normal myotubes. a Immunoblot analysis of IK from C2C12 myoblasts transfected with silK for $48 \mathrm{~h}$. b Relative mRNA levels of IK from C2C12 myoblast transfected with silK for $48 \mathrm{~h}$ using qRT-PCR analysis. $18 \mathrm{~S}$ rRNA was used as a normalization control. ***p <0.001. c Immunoblot analysis of Pax7, MyoD, and MyoG from C2C12 myoblasts transfected with silK for $48 \mathrm{~h}$. $\mathbf{d}$ Immunocytochemistry image stained with anti-MYH antibody from IK-depleted C2C12 cells at day 3 of differentiation. Scale bar $=20 \mu \mathrm{m}$ (left panel). For fusion index for myotubes, the ratio of the number of nuclei in $\mathrm{MYH}$-positive myotubes per the total nuclei in one field was quantified from four random microscopic fields; (right panel) ${ }^{* *} p<0.001$. e Immunoblot analysis of MyoG and MYH from C2C12 cells transfected for $18 \mathrm{~h}$ and harvested at days $0,1,2$, and 3 after differentiation (left panel). The band intensity normalized to $\beta$-actin was graphed by ImageJ software (right panel). ${ }^{* *} p<0.01,{ }^{* * *} p<0.001$. $\mathbf{f}, \mathbf{g}$ Relative mRNA levels of MyoD and MyoG from C2C12 myoblast $\mathbf{f}$ at day 2 and $\mathbf{g}$ day 3 after differentiation using qRT-PCR analysis. 185 rRNA was used as a normalization control. ${ }^{*} p<0.05,{ }^{* *} p<0.01,{ }^{* * *} p<0.001$

reciprocal cell transplantation between WT and $i k \mathrm{MO}$ embryos to determine whether $i k$ acts in a cellautonomous or non-cell-autonomous manner in muscle development. First, we injected $i k$ morpholino into $T g$ (mito: GFP) donor embryos harboring transgenic mitochondria-targeted green fluorescent protein (mitoGFP) at the one-cell stage. Next, the green fluorescent $i k$ MO donor cells were transplanted into WT host embryos at 4 hpf (Fig. 6a). At 36 hpf, we observed the expression of GFP in a chimeric WT host embryo using fluorescence microscopy (Fig. 6b). In the skeletal muscle position, the chimeric GFP-positive muscle cells showed a WT muscle phenotype. Furthermore, as we previously observed that fast-twitch muscle fibers are impaired in $i k \mathrm{KO}$ embryos, fast-twitch muscle fibers of the chimeric WT host embryos at $36 \mathrm{hpf}$ were observed using wholemount immunohistochemistry (Fig. 6c). The chimeric GFP-positive muscle cells stained with anti-GFP and F310 antibodies, to detect transplanted green fluorescent cells and fast-twitch muscle fibers, respectively, exhibited a normal phenotype in fast-twitch muscle fibers consistent with that observed in WT embryos at $36 \mathrm{hpf}$ (Fig. $6 \mathrm{~d})$. In the reciprocal experiment, $i k \mathrm{MO}$ was injected into WT host embryo at the one-cell stage and the green fluorescent WT $\mathrm{Tg}$ (mito: GFP) donor cells were transplanted into the $i k \mathrm{MO}$ host embryo at $4 \mathrm{hpf}$ (Fig. 6e). At $36 \mathrm{hpf}$, we observed the GFP expression in a chimeric $i k$ MO host embryo at 36 hpf (Fig. 6f). The GFP-labeled cells located in the skeletal muscle showed less fused myoblasts and failed to form normal myotubes. Using whole-mount immunohistochemistry with anti-GFP and anti-F310 (Fig. 6g), we observed that the transplanted chimeric GFP-positive muscle cells did not display a normal muscle fiber, similar to impaired skeletal muscles in $i k$ MO embryos (Fig. 6h). Collectively, these results support that IK functions in a non-cell-autonomous manner in zebrafish muscles.

\section{Myoblast proliferation and apoptosis was not affected in ik KO embryos}

To rule out the possibility that the decrease in myoblast differentiation was due to proliferation inhibition and/or an increase in apoptosis, myoblast proliferation impairment and/or apoptosis was examined in $i k \mathrm{KO}$ embryos.
First, BrdU cell proliferation assay was performed to compare cell proliferation between WT and $i k \mathrm{KO}$ embryos. There was no significant change in the number of BrdU-positive cells between WT and $i k \mathrm{KO}$ embryos (Fig. 7a). Furthermore, acridine orange staining for screening apoptotic cells in living embryos was almost undetectable in the posterior region of $i k \mathrm{KO}$ embryos until $5 \mathrm{dpf}$ and slightly increased in the brain and heart after $5 \mathrm{dpf}$ (Fig. 7b). Next, we examined whether loss of IK affects the apoptosis of myoblast cells. No significant changes were observed in IK-depleted $\mathrm{C} 2 \mathrm{C} 12$ myoblasts in poly (ADP-ribose) polymerase (PARP), caspase-3, and caspase-9, which are common apoptosis markers (Fig. 7c). We confirmed that IK is not involved in the maintenance of self-renewing myoblasts and apoptotic cell death. Consequently, our results indicate that loss of IK disrupts myogenic transcription factors for myogenesis, resulting in impaired muscle development and embryonic lethality.

\section{Discussion}

Alternative pre-mRNA splicing produces diverse protein isoforms from one gene and contributes to various functions $[3,26,27]$. Because alternative pre-mRNA splicing affects normal biological development and phenotypic complexity in most mammals, pre-mRNA splicing defects or dysregulation causes several diseases including cancer, neurodegenerative diseases, and muscular dystrophy $[28,29]$. IK, one of the splicing factors, is known to play an essential role during the activation of the spliceosome B complex by mediating the interaction of multiple proteins in the spliceosome [12, 30]. Specifically, IK is structurally important in the spliceosome by bridging $\mathrm{U} 2$ with U5 proteins in the B complex, as deduced from the 3D cryogenic electron micrographs of the precatalytic human B complex [31]. Recently, IK has been reported to alleviate structural constraints that prevent the activation of spliceosomes formed on introns with a short 5'SS-BS distance during spliceosome activation in HeLa cells [14]. Besides, IK participates in a variety of cellular processes, such as cell cycle progression [11] and genome stability [32], and regulates influenza virus gene expression by binding to the viral RNA polymerase [13]. Although the role of IK has been studied previously 
A

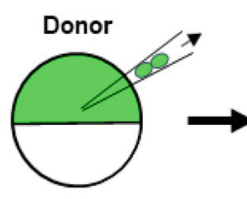

ik MO

Tg(mito:GFP)
B

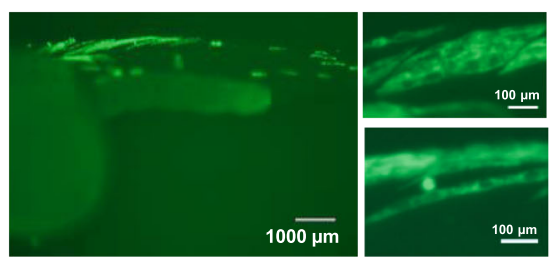

D

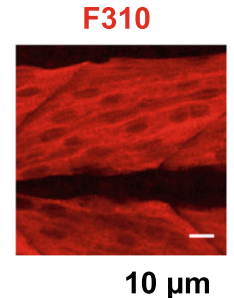

E

F
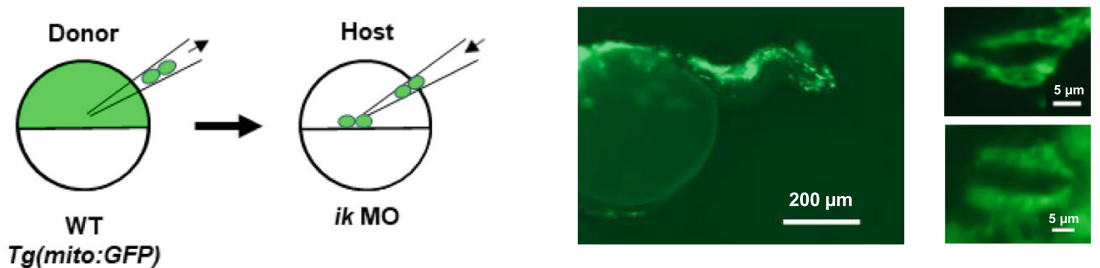

G

H

DAPI

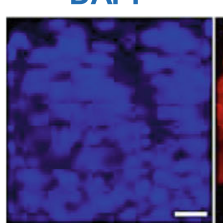

F310

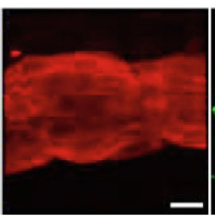

GFP

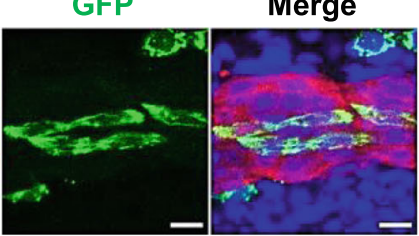

$10 \mu \mathrm{m}$

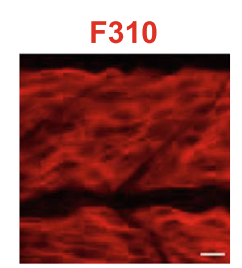

$10 \mu \mathrm{m}$

Fig. $6 \mathrm{IK}$ functions in a non-cell-autonomous manner in muscle precursors in zebrafish. a Schematic diagram of the cell transplantation. ik morpholino (MO) is injected into Tg (mito: GFP) donor embryo at the one-cell stage. At 4 hpf, the green fluorescent ik MO donor cells are transplanted into WT host embryo. b GFP expression in a chimeric WT host embryo at $36 \mathrm{hpf}$ (left panel). High magnification image of the GFPlabeled cells located in the skeletal muscle (right panel). c Confocal images of chimeric WT host embryo at 36 hpf stained with anti-F310 antibody for fast-twitch muscle fibers and anti-GFP antibody for green fluorescent cells transplanted from ik MO donor embryos. $\mathbf{d}$ Confocal images of fast-twitch muscle fibers of WT embryos at $36 \mathrm{hpf}$ stained with anti-F310 antibody. e Schematic diagram of cell transplantation. ik MO is injected into WT host embryo at the one-cell stage. At 4 hpf, the green fluorescent WT Tg (mito: GFP) donor cells are transplanted into ik MO host embryo. f GFP expression in a chimeric ik MO host embryo at 36 hpf (left panel). High magnification image of the GFP-labeled cells located in the skeletal muscle (right panel). $\mathbf{g}$ Confocal images of chimeric ik MO host embryo at $36 \mathrm{hpf}$ stained with anti-F310 antibody for fast-twitch muscle fibers and anti-GFP antibody for green fluorescent cells transplanted from WT donor embryo. $\mathbf{h}$ Confocal images of fast-twitch muscle fibers of ik MO embryo at 36 hpf stained with anti-F310 antibody

in vitro, little is known about how IK functions in vivo. Here, we generated the $i k \mathrm{KO}$ vertebrate animal model through CRISPR/Cas9 technology in zebrafish and studied the function of IK.
The major difference of CRISPR/Cas9-mediated $i k \mathrm{KO}$ embryos compared to WT is that they exhibit severe body deformities such as downward tail curvature. Intriguingly, we have found that $i k \mathrm{KO}$ embryos show impaired NMJ which could be caused by neural or 
A
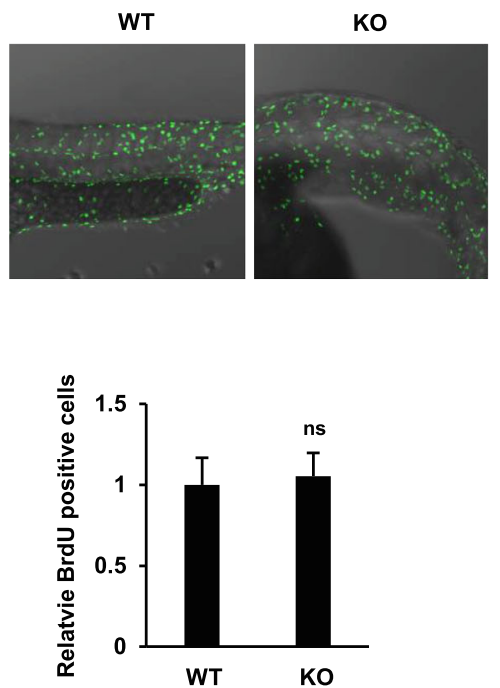

B

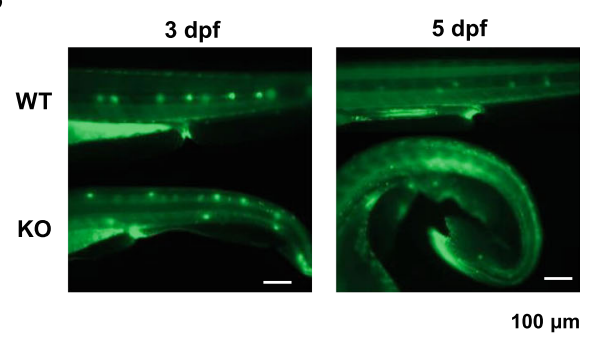

C

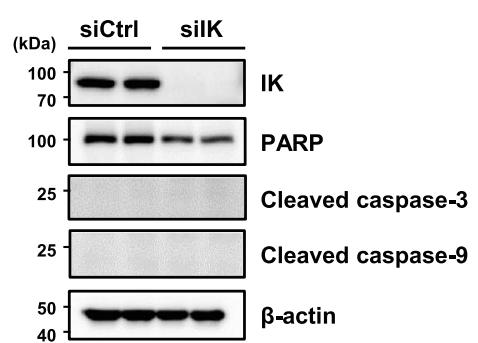

Fig. 7 Myoblast proliferation and apoptosis was not affected in ik KO embryos. a Comparison of cell proliferation estimated through BrdU incorporation in $3 \mathrm{dpf}$ WT and ik KO embryos. The relative number of BrdU-positive cells shown in the graph. ns not significant. b Analysis of apoptosis in 3 and $5 \mathrm{dpf}$ WT and ik KO live embryos with acridine orange staining. c Immunoblot analysis of PARP, cleaved caspase-3, and cleaved caspase-9 in C2C12 myoblasts transfected with silk\#1 for $48 \mathrm{~h}$

muscular defects. Indeed, abnormal skeletal muscle phenotype was identified in $i k \mathrm{KO}$ embryos, supporting that IK affects muscles rather than neurons. Furthermore, it was confirmed that IK has a non-cellautonomous function in skeletal muscle development. This suggests that the loss of IK in the skeletal muscle is likely to contribute to abnormal muscle morphology in a non-cell-autonomous manner. Meanwhile, although a previous study has reported that $i k$ MO embryos exhibit myocardial contractile dysfunction in zebrafish [33], $i k$ MO embryos did not show a significant difference in cardiac structure compared to WT (Additional file 4: Video S3). Given the abnormal skeletal muscle phenotype, such as downward tail curvature, appeared from $1.5 \mathrm{dpf}$ in $i k \mathrm{KO}$ embryos, the loss of $i k$ might be fatal for long-term maintenance of tissue development rather than for initial formation. Thus, more specific effects of IK should be studied depending on the stage or site of embryonic development for future research.

In addition, RNA-seq analysis revealed that skeletal muscle differentiation genes are downregulated in $i k \mathrm{KO}$ embryos. Especially, 34\% transcripts of fast muscle genes were downregulated based on GO enrichments in $i k \mathrm{KO}$ embryos compared to WT (data not shown). Thus, taking into consideration that IK plays a critical role in splicing where the intron length is less than $200 \mathrm{bp}$ [14], we confirmed splicing events of myogenesis-related genes with primers designed to amplify exons covering short introns $(<200 \mathrm{bp})$. In conclusion, several genes, mainly expressed in fast-twitch muscle fibers such as mybpc2a and smyd1a, showed abnormal splicing patterns, suggesting a possibility that improper splicing events in fast-twitch muscle fiber genes might induce impairment of fast-twitch muscles. However, despite being in the $i k$ $\mathrm{KO}$ embryos, certain exons encompassing small-sized introns such as mybpc1 E19-E20, tnnt2e E9-E10, acta1a E7-E8, and acta1a E15-E16 have been found to be precisely spliced (Fig. 3h, j, l). According to Keiper et al. [14], most of the introns maintained after knockdown of IK are predominantly shorter than $200 \mathrm{nts}$, but a substantial fraction of longer introns (30-40\%) exists. The authors suggest that the $5^{\prime}$ SS-BS distance might be a crucial factor for activation of IK rather than the intron length. Thus, splicing of muscle genes having short introns in the absence of IK could be caused by the long distance between $5^{\prime}$ SS and BS. However, it is still ambiguous whether impaired fast-twitch muscle fibers in $i k$ $\mathrm{KO}$ embryos are definitively caused by altered splicing events because all fast muscle transcripts were not downregulated. Furthermore, downregulation of mRNA transcript can also occur due to many mRNA degradation mechanisms including instability of mRNA transcripts during RNA processing, increased nonsensemediated mRNA decay (NMD) or non-stop decay (NSD), and secondary transcription-level processes [3436]. Our findings that IK plays a non-cell-autonomous role in muscle in the transplant experiments suggest that perhaps other signaling extrinsic factors may be partially involved in splicing events mediated by IK in vivo. Similarly, this can also be supported by the fact that the $i k$ 
$\mathrm{KO}$ embryos and IK-deficient myoblasts were not affected by apoptosis (Fig. 7), although knockdown of IK has been reported to induce apoptosis in cancer cells [10]. In other words, considering there are other differentially expressed genes as well as muscle-related genes among the diverse biological process categories in the RNA-seq data (Fig. 2a), further studies are required to determine specific splicing events and regulatory mechanisms of transcription networks associated with IK at various tissue types or stages of development.

Muscle is one of the tissues wherein pre-mRNA splicing plays a key role in reprogramming of gene transcripts [37]. Thus, splicing factors involved in muscle development tightly control pre-mRNA splicing of muscle-specific genes, and alteration of these splicing factors leads to diverse muscle disorders. There are many representative splicing factors known to be related in muscle differentiation including RNA binding motif protein 20 (RBM20), RBM24, RBM4, polypyrimide tract binding protein (PTB), RNA binding protein, and fox-1 homolog [38-41]. For example, one of the major muscle-specific splicing factors, RBM20, consists of two domains including an RNA-recognition domain 1 and a serine (S)/arginine (R)-rich domain required for spliceosome assembly [42, 43]. As RBM20 functions in the premRNA splicing of the TTN gene, which provides connections at the level of individual microfilaments, loss of RBM20 inhibits muscle differentiation and leads to heart diseases such as cardiomyopathy and ischemic heart disease in vivo $[44,45]$. In this study, we identified that splicing factor IK is involved in the splicing event of muscle-specific genes and contributes to normal muscle development. Although more research is needed, our findings suggest that regulating the levels of IK in muscle cells could be a therapeutic approach for a variety of muscle degenerative diseases.

On the other hand, during myogenesis, myoblasts are first activated by MRFs and begin to differentiate to form muscle fibers [46]. In particular, the pairedhomeobox transcription factors, Pax3 and Pax7, regulate the initiation of muscle differentiation by promoting the expression of MRFs of the MyoD family [47]. MyoD operates as a myogenic determinant and is involved in terminal differentiation of skeletal myoblasts. Previous studies have described the distribution and expression of Pax7 and MyoD with multiple spliced regions/domains in various species [48-52]. While we found an upregulated $\operatorname{pax} 7 a$ and downregulated $\operatorname{myod} 1$ at mRNA level in $i k \mathrm{KO}$ embryos and IK-depleted $\mathrm{C} 2 \mathrm{C} 12$ cells, whether these proteins interact directly and how they function in vivo is still largely obscure. As an endogenous IK interacts with endogenous Pax7 and MyoD in C2C12 cells, IK protein function might be associated with myogenic differentiation (Additional file 10: Figure S5). It suggests that molecular interactions between IK and MRFs mediate signaling cross-talk for the myogenesis in the skeletal muscle in vivo.

\section{Conclusion}

In conclusion, we found that loss of IK leads to embryonic defects in zebrafish, including impairment of fasttwitch muscle fibers, and altered pre-mRNA splicing of skeletal muscle differentiation genes. Furthermore, IKdepleted $\mathrm{C} 2 \mathrm{C} 12$ myoblasts had a reduced ability to form myotubes during myogenesis compared to WT. Thus, we propose that splicing factor IK contributes to normal skeletal muscle development.

\section{Materials and methods}

\section{Zebrafish lines}

WT AB* transgenic $T g$ (mito-GFP), and $T g$ (kdrl: GFP) zebrafish (Danio rerio) were used in this study. Embryos/larvae were raised at $28.5^{\circ} \mathrm{C}$ and staged in hpf or $\mathrm{dpf}$ as per a previously described standard procedure [53].

\section{Generation of $i k$ KO zebrafish}

Single guide RNA (sgRNA) target site in the CRISPR system was designed for IK exon2 using the ZiFiT Targeter website program to identify the sgRNA sequences with high on-target activity: 5'-GGCTCCAGATGGCC ATGAGG-3' [54, 55]. Gene-specific oligonucleotides for sgRNA were produced using the PCR-based short-oligo method followed by a 20-base target sequence without the PAM (Marcrogen; Seoul, Korea). sgRNAs were synthesized in vitro from purified PCR products using mMESSAGE mMACHINE T7 RNA transcriptase kit (Thermo Fisher Scientific). To synthesize Cas9 protein, pET-NLS-Cas9-6xHis was purchased from Addgene (USA, plasmid \#62934) and purified as described previously [56]. $i k$-gRNA and Cas9 protein were co-injected in the one-cell stage $A B^{*}$ zebrafish embryos. Injected embryos were grown to adulthood and screened for germline transmission of CRISPR-induced mutations. For PCR amplification of the $i k$ locus from F2 larvae, genomic DNA was used as the template and amplified with primers (forward: 5' -GTGAGCATGTAACAAGTA CT-3'/reverse: 5' -CATATTAAGTCGGGATAGTC-3'). The PCR product was confirmed by digestion with $B s l$ or sequenced by BIONICS Inc. (Korea).

\section{RNA-seq}

For RNA-seq, the $i k$ phenotype was examined in $i k$ gene-targeted G5 embryos from heterozygote incrosses within individual $i k$ G4 lines. All embryos in a clutch were scored for $i k$ phenotypes (curved tail and slowed heartbeat morphology), wherein the phenotype was detected in 39/164 (23.7\%) $i k$ nulls and 123/164 (75\%) 
wild-types/heterozygotes from 4 different crosses. The 20 embryos were randomly selected among G5 embryos in $i k$ nulls and wild-types/heterozygotes from individual 4 different crosses and genotyped by sequencing (100\% match for phenotypes). $i k$ phenotype was $100 \%$ linked to the $i k$ genotype. We performed RNA-seq using 50 G5 samples of WT and mutant embryos at $3 \mathrm{dpf}$.

Total RNA was extracted from samples using TRIzol reagent (Invitrogen) according to the manufacturer's protocol and confirmed to have an absorbance ratio $>$ 1.8 and integrity $>7.0$. The total mRNA-seq was performed by eBiogen (Seoul, Korea). Libraries were prepared from total RNA using the SMARTer Stranded RNA-Seq Kit (Clontech Laboratories) and the isolation of mRNA was performed using the Poly(A) RNA Selection Kit (LEXOGEN). The isolated mRNAs were used for the cDNA synthesis and indexed using the Illumina indexes 1-12. The enrichment step was performed using PCR. Subsequently, libraries were checked using the Agilent 2100 bioanalyzer (DNA High Sensitivity Kit) to evaluate the mean fragment size. Then, high-throughput sequencing was performed as paired-end 100 sequencing using HiSeq 2500 (Illumina), yielding $>4$ Gb data/sample with a 40x read depth. After sequencing, a quality control of raw sequencing data was performed and lowquality reads were removed. Next, Fragments Per Kilobase of transcript per Million reads (FPKM) were used to determine the expression levels of the genes. Upregulated or downregulated genes were identified using ExDEGA v1.6.0 (eBiogen, Korea) and categorized based on a search performed using DAVID (http://david.abcc. ncifcrf.gov). Approximately 1500 genes selected from the differentially expressed gene (DEG) analysis were analyzed by DAVID. The GO from the DAVID analysis was used to show the correlation in Quick GO (https://www. ebi.ac.uk/QuickGO). The clustering heatmap profiles of DEGs were analyzed according to similarities in gene function using the Multiple Experiment Viewer software program $\mathrm{v} 4.9(\mathrm{MeV})$. A gene set representing $>2$-fold changes in $i k \mathrm{KO}$ zebrafish was presented by hierarchical clustering analysis (red, > 2-fold change; blue, <2-fold change).

To visualize splicing pattern of genes, Sashimi plots were generated using Integrative Genomics Viewer (IGV) $[24,57]$. In brief, the RNA-seq read alignments of the sample in BAM file format are loaded in the IGV browser and the isoform expression levels of transcripts are estimated by the MISO algorithm [58]. Then, Sashimi plots showing splicing patterns for the genomic region are presented. In Sashimi plots, genomic location is shown on the $x$-axis and genomic read density is expressed on the $y$-axis. In each plot, junction reads are indicated as the "bridges" with the numbers of junction read counts on the bridges.

\section{Whole-mount in situ hybridization}

The riboprobes were generated from $\operatorname{pax} 7 a$ and $\operatorname{myod} 1$ encoded pCS2+ plasmid, which was linearized with BamH1 (Elpis). T7 RNA polymerase (Ambion) was used for in vitro RNA transcription and the riboprobes were labeled with digoxigenin using DIG RNA labeling Kit (Roche). Whole-mount in situ hybridization was performed using previously described standard protocols [59]. The resulting images were captured by a camera attached to a microscope (Nikon SMZ1500).

\section{Morpholino oligonucleotide microinjection and cell transplantation}

The sequence for $i k$ morpholino oligonucleotides is $5^{\prime}$ GGAGCCAGAGGATTAGAGTACACAT-3', as previously described [33], and was purchased from GeneTools (Corvallis, OR). For knockdown of $i k, 4 \mathrm{ng}$ of $i k$ $\mathrm{MO}$ was injected into fertilized zebrafish egg at the onecell stage. For transplantation, WT or $i k$ MO embryos at mid-blastula stages were dechorionated with $0.5 \mathrm{mg} / \mathrm{ml}$ pronase (Roche) and cell transplantation was performed according to the protocol described previously [60]. Chimeric embryos formed by transplantation were fixed at $36 \mathrm{hpf}$ and imaged using a fluorescence microscope (Zeiss Axio Zoom V16) and LSM-700 confocal laser scanning microscope (Carl Zeiss).

\section{Detection of cell proliferation and apoptosis in zebrafish}

To detect cell proliferation, dechorionated zebrafish embryo was chilled on ice in egg water for $15 \mathrm{~min}$ and incubated on $10 \mathrm{mM}$ BrdU/15\% dimethylsulfoxide (DMSO) solution (Sigma-Aldrich) at different time points to allow BrdU uptake. The embryos were then fixed with $4 \%$ paraformaldehyde for $2 \mathrm{~h}$ at $26^{\circ} \mathrm{C}$, digested with $10 \mu \mathrm{g} /$ $\mathrm{ml}$ Proteinase K (Thermo Fisher Scientific) for $10 \mathrm{~min}$, incubated in $2 \mathrm{~N} \mathrm{HCl}$ for $1 \mathrm{~h}$, and rinsed with PBST [1× phosphate-buffered saline (PBS), 0.1\% Triton X-100]. For BrdU staining, they were blocked with BrdU blocking solution (10\% goat serum in PBST) for $30 \mathrm{~min}$, incubated with primary mouse monoclonal anti-BrdU (Abcam, 1:100) overnight, and treated with goat antimouse IgG $(\mathrm{H}+\mathrm{L})$ secondary antibody (Alexa Fluor 488, Thermo Fisher Scientific) for visualization. The BrdUpositive nuclei were counted using an LSM-700 confocal laser scanning microscope (Carl Zeiss). To detect apoptotic cells in live zebrafish embryos, zebrafish embryos were dechorionated and soaked in egg water containing the vital dye, acridine orange $(2 \mu \mathrm{g} / \mathrm{ml})$, at $28^{\circ} \mathrm{C}$ for 30 min. After washing with egg water, zebrafish were anesthetized with tricaine, mounted in $2 \%$ methylcellulose, and examined with a fluorescence microscope (Zeiss Axio Zoom V16). 


\section{Cell culture}

C2C12 myoblasts were cultured in Dulbecco's modified Eagle's medium (DMEM) supplemented with $15 \%$ fetal bovine serum (GE Healthcare Life Science) at $37^{\circ} \mathrm{C}$ in a humidified atmosphere of $5 \% \mathrm{CO}_{2}$. For induction of differentiation, fully confluent $\mathrm{C} 2 \mathrm{C} 12$ myoblasts were cultured in DMEM supplemented with $2 \%$ horse serum (Sigma-Aldrich) for 3 days.

\section{Antibodies}

The primary antibodies used for immunoblotting and immunofluorescence were as follows: rabbit polyclonal anti-IK (Bethyl Laboratories, A301-708A), mouse monoclonal anti- $\beta$-actin (Santa Cruz, sc-47778), mouse monoclonal anti-Pax-7 (Santa Cruz, sc-81648), mouse monoclonal anti-MyoD (Santa Cruz, sc-377460), mouse monoclonal anti-myogenin (Santa Cruz, sc-52903), rabbit polyclonal anti-MyHC (Santa Cruz, sc-20641), mouse polyclonal anti-PARP (Cell Signaling, \#9542), rabbit polyclonal anti-Cleaved Caspase-3 (Asp175) (Cell Signaling, \#9661), rabbit monoclonal anti-Cleaved Caspase-9 (Asp315) (Cell Signaling, \#20750), anti-GFP (Cell Signaling, \#2956), anti-Bungarotoxin Alexa Fluor ${ }^{\text {tw }}$ 647 conjugate (Invitrogen ${ }^{\text {ra }}$, \#B35450), anti-fast-twitch muscle (DSHB, F310), and anti-slow-twitch muscle (DSHB, F59). The horseradish peroxidase-conjugated goat anti-mouse or anti-rabbit IgG (Fab) secondary antibodies were purchased from Enzo Life Sciences.

\section{siRNA transfection}

siRNA was transfected into $\mathrm{C} 2 \mathrm{C} 12$ cells using Lipofectamine RNAiMax Transfection Reagent (Invitrogen) according to the manufacturer's transfection protocol. The following siRNA oligonucleotides synthesized by Bioneer were used to suppress IK: 5'-CUGCAAAAGAGUUG AUCAA-3'. Final siRNA concentration was adjusted at $20 \mathrm{nM}$ and incubated for $48 \mathrm{~h}$ after siRNA transfection.

\section{Immunofluorescence}

$\mathrm{C} 2 \mathrm{C} 12$ cells grown on coverslips were immediately permeabilized with $0.1 \%$ Triton X-100 in $1 \times$ PBS for 3 min and subsequently fixed with $4 \%$ paraformaldehyde in PBS for $10 \mathrm{~min}$. The cells were then washed twice with PBS and blocked with PBS-BT (3\% bovine serum albumin or BSA and $0.1 \%$ Triton X-100 in PBS) for 15 min at $26^{\circ} \mathrm{C}$. The cells on the coverslips were subsequently incubated with primary antibody (anti-MyHC, 1 : 200) and then with tetramethylrhodamine (TRITC)coupled secondary antibody $(1: 500)$ for $1 \mathrm{~h}$ at $26^{\circ} \mathrm{C}$. The nuclei of the fixed cells were stained with $4^{\prime}, 6^{\prime}$-diamidino-2-phenylidole (DAPI) mounting medium. Images were acquired on a confocal microscope and analyzed using ZEN software (Nikon).

\section{Immunohistochemistry}

For whole-mount immunostaining of zebrafish embryo, embryos were fixed in $4 \%$ paraformaldehyde in PBS at $4{ }^{\circ} \mathrm{C}$ overnight and blocked with blocking solution (1× PBS, 3\% BSA, $1 \%$ Triton X-100) for $2 \mathrm{~h}$ at $26^{\circ} \mathrm{C}$. After washing with $1 \times$ PBS for $30 \mathrm{~min}$, the fixed embryo was incubated with primary antibodies (F310: 1:10, F59: 1:2, GFP: 1:400, BTX: 1:300) diluted in blocking solution at $4{ }^{\circ} \mathrm{C}$ overnight. Next, it was washed three times in $1 \times$ PBS with $1 \%$ Triton X-100 for 30 min each. After, samples were incubated with fluorescein isothiocyanate (FITC)- or TRITC-coupled secondary antibodies (1:500) diluted in blocking solution overnight at $4{ }^{\circ} \mathrm{C}$. After washing with $1 \times$ PBS for $30 \mathrm{~min}$, images were acquired using a confocal microscope (Zeiss Axio Zoom V16). For transverse sections, fixed embryos were incubated in $30 \%$ sucrose solution at $4{ }^{\circ} \mathrm{C}$ overnight. Next day, the embryos were covered with cryo-embedding media (OCT compound) in mold and contained into liquid nitrogen until the mold block was completely frozen. For sectioning, the frozen embryo block was transferred to the cryotome and sectioned into $15-\mu \mathrm{m}$-thick sections. The tissue section placed onto the glass slide was dried at $26^{\circ} \mathrm{C}$ overnight and stained with antibody, as described for whole-mount immunostaining.

\section{Immunoblot analysis}

The cells were lysed in lysis buffer [50 mM Tris- $\mathrm{HCl}$ (pH 8.0), $150 \mathrm{mM} \mathrm{NaCl}, 1 \mathrm{mM}$ EDTA, $1 \% \mathrm{NP}-40$, a protease and phosphatase inhibitor mixture (Roche)] and centrifuged for $15 \mathrm{~min} 20,000 \times g$ at $4{ }^{\circ} \mathrm{C}$ to obtain the cell lysates. Next, the cell lysate concentration was quantified using the Pierce BCA Protein Assay Kit (Thermo Scientific). The total protein sample was prepared using $5 \times$ sodium dodecyl sulfate (SDS) sample buffer and heated at $99^{\circ} \mathrm{C}$ for $10 \mathrm{~min}$. Proteins were separated on a $10 \%$ SDS-polyacrylamide electrophoresis gel and transferred to a $0.45-\mu \mathrm{m}$ pore size nitrocellulose membrane (GE Healthcare Life Science). The membrane was incubated overnight at $4{ }^{\circ} \mathrm{C}$ with primary antibodies in TBS-T [150 $\mathrm{mM} \mathrm{NaCl}, \quad 20 \mathrm{mM}$ Tris- $\mathrm{HCl}$ (pH 8.0), and $0.05 \%$ Tween-20] containing 3\% BSA, followed by secondary antibody incubation using horseradish peroxidaseconjugated goat anti-mouse or anti-rabbit IgG (Fab) (Enzo Life Sciences) in 5\% skim milk dissolved in TBS-T at $26^{\circ} \mathrm{C}$ for $2 \mathrm{~h}$. Proteins were visualized with an ECL western blotting reagent (BioNote) and analyzed on a Fusion Solo-S image analyzer (Vilber). Protein band intensities were quantified and analyzed using ImageJ software.

\section{Immunoprecipitation assay}

For the immunoprecipitation assay, the cells were lysed with lysis buffer and centrifuged for $15 \mathrm{~min}$ at $4{ }^{\circ} \mathrm{C}$ at 20 , 
$000 \times g$ to obtain the cell lysates, which were incubated with $2 \mu \mathrm{g}$ antibody for $2 \mathrm{~h}$ at $26^{\circ} \mathrm{C}$. This step was followed by incubation with protein $\mathrm{G}$ agarose beads (Amicogen, 2010005) overnight at $4{ }^{\circ} \mathrm{C}$. We used normal rabbit IgG (Santa Cruz, sc-2027) as a negative control antibody. Then, the immunocomplexes were washed with lysis buffer five times, and the immunocomplexes were separated by SDS-polyacrylamide gel and detected by immunoblotting analysis.

\section{PCR and quantitative RT-PCR}

Total RNA was extracted using TRIzol (Takara) following the manufacturer's instructions. After RNA extraction, the total RNA was measured using an Epoch2 microplate spectrophotometer (BioTek) and $3 \mu \mathrm{g}$ RNA sample was reverse transcribed into cDNA using MMLV Reverse Transcriptase (Thermo Scientific). For standard PCR, AccuPower PCR PreMix (Bioneer) was used. The qRT-PCR was performed using Maxima SYBR Green (Thermo Scientific) with an ABI7500 or a Quantstudio 3 real-time PCR detection system (Applied Biosystems). The primer sequences for PCR and qRT-PCR are provided in Additional file 11: Table S3. Reaction specificity was confirmed by melting curve analysis and the comparative $C_{\mathrm{t}}$ method was used to analyze relative gene expression. The $18 \mathrm{~S}$ rRNA was used to normalize the results in the delta-delta $\mathrm{Ct}$ analysis.

\section{Statistical analysis}

Values are presented as mean \pm standard deviation (SD). Multiple comparisons within groups were performed by one-way analysis of variance (ANOVA), and differences between the means of individual groups were evaluated using the Student's $t$ test. A value of $p<0.05$ was considered as the threshold for significant differences $(* p<$ $0.05,{ }^{* * *} p<0.01,{ }^{* * * *} p<0.001$ ).

\section{Supplementary Information}

The online version contains supplementary material available at https://doi. org/10.1186/s12915-021-00980-y.

Additional file 1: Figure S1. The homology of IK between human and zebrafish. A sequence alignment of $\mathbb{I K}$ cytokine proteins from human and zebrafish by NCBI COBALT. The conservation is shown below in red.

Additional file 2: Video S1. The heart of WT and ik KO embryo at 2 days post-fertilization. Heartbeat of WT (left) and ik KO embryos (right) at 2 days post-fertilization.

Additional file 3: Video S2. The heartbeat of $\mathrm{WT}$ and $i k \mathrm{KO}$ zebrafish embryos at 3 days post-fertilization. Heartbeat of WT (upper) and ik KO embryos (lower) at 3 days post-fertilization.

Additional file 4: Video S3. The heart of $T g$ ( $k d r l:$ GFP) WT and ik MO zebrafish embryos. The heart of the $T g$ (kdrl: GFP) wild type zebrafish, where cardiomyocytes express GFP in a vascular-specific manner (upper) and ik MO-injected embryos (lower) at $48 \mathrm{~h}$ post-fertilization. The position of the heart is indicated by the white arrow.
Additional file 5: Table S1. The raw data of RNA-seq for skeletal muscle differentiation genes listed in Fig. 2c.

Additional file 6: Figure S2. The Sashimi plots of skeletal muscle differentiation genes in RNA-seq. Sashimi plots of skeletal muscle differentiation genes, including (A) cdkn1a, (B) mybpc2a (C) mybpcl, (D) tnntze, (E) smydla, (F) tnni3k, and (G) actala from the Integrative Genomics Viewer (IGV) browser in WT (blue plots; lower) and ik KO embryos (red plots; upper). In each plot, minimum splice junction coverage was set to 5 for visual clarity and statistical significance.

Additional file 7: Figure S3. The Sashimi plots of housekeeping genes in RNA-seq. Sashimi plots of housekeeping genes including (A) actb1, (B) gapdh, and (C) tubalb from the Integrative Genomics Viewer (IGV)

browser in WT (blue plots; lower) and ik KO embryos (red plots; upper). In each plot, minimum splice junction coverage was set to 5 for visual clarity and statistical significance.

Additional file 8: Table S2. The raw data of myod1 and pax7a in RNAseq.

Additional file 9: Figure S4. Sashimi plots of pax7a in RNA-seq. Sashimi plots of pax7a gene from the Integrative Genomics Viewer (IGV) browser in WT (blue plots; lower) and ik KO embryos (red plots; upper). Minimum splice suction coverage was set to 5 for visual clarity and statistical significance.

Additional file 10: Figure S5. The immunoprecipitation of IK with MyoD and Pax7 in $\mathrm{C2} 12$ cells. Immunoblot analysis of MyoD and Pax7 after endogenous immunoprecipitation using an anti-IK antibody from C2C12 myoblasts. As a negative control antibody, anti-normal rabbit lgG antibody was used.

Additional file 11: Table S3. List of primer sequences used for RT-PCR analysis.

Additional file 12. Uncropped images of immunoblots in figures. The raw immunoblots of the membranes in Fig. 5a, c, e, 7c and Figure S5 are presented.

\section{Acknowledgements}

We are grateful to Ga Eun Chung, Woo Jung Kim, and Gaeun Oh for supporting experiments and providing assistance in the laboratory work.

\section{Authors' contributions}

HIK planned the research, performed the experiments, and wrote the manuscript. HS, YC, JK, MC, and SYC conducted the in vivo experiments and interpreted the RNA-seq data. SP and SH performed the in vitro experiments. JA and HSC designed the CRISPR/Cas9 system and analyzed the data. YY and MJK supervised the entire project, co-wrote, and proofread the manuscript. All authors read and approved the final manuscript.

\section{Funding}

This research was supported by the National Research Foundation of Korea (NRF) grant funded by the Korean government (2016R1A5A1011974, 2017R1D1A1B03033153, 2020R1A2C2100985, 2021R1A2C3003414) and the University Innovation Support Project of Sookmyung Women's University Research Grants.

\section{Availability of data and materials}

All data generated or analyzed during this study are included in this published article and its additional information files.

\section{Ethics approval and consent to participate}

All zebrafish husbandry and experimental protocols complied with institutional guidelines and were approved by local ethics boards (Sookmyung Women's University Animal Care and Use Committee, SMWUIACUC-1712-036).

\section{Consent for publication}

Not applicable

\section{Competing interests}

The authors declare no competing interests. 


\section{Author details}

'Department of Biological Sciences, Sookmyung Women's University, Seoul 04310, Republic of Korea. ${ }^{2}$ Research Institute of Women's Health, Sookmyung Women's University, Seoul 04310, Republic of Korea. ${ }^{3}$ Howard Hughes Medical Institute and Department of Molecular Biosciences, University of Texas at Austin, Austin, TX 78712, USA. ${ }^{4}$ Department of Biomedical Sciences, Chonnam National University Medical School, Hwasun 58128, Republic of Korea. ${ }^{5}$ Center for Theragnosis, Biomedical Research Institute, Korea Institute of Science and Technology (KIST), Seoul 02792, Republic of Korea. ${ }^{6}$ Division of Bio-Medical Science and Technology, KIST School, University of Science and Technology (UST), Seoul 02792, Republic of Korea.

Received: 17 December 2020 Accepted: 11 February 2021 Published online: 01 April 2021

\section{References}

1. Brow DA. Allosteric cascade of spliceosome activation. Annu Rev Genet. 2002;36:333-60

2. Wahl MC, Will CL, Luhrmann R. The spliceosome: design principles of a dynamic RNP machine. Cell. 2009;136:701-18.

3. Lee $Y$, Rio DC. Mechanisms and regulation of alternative pre-mRNA splicing. Annu Rev Biochem. 2015;84:291-323.

4. Shi Y. Mechanistic insights into precursor messenger RNA splicing by the spliceosome. Nat Rev Mol Cell Biol. 2017;18:655-70.

5. Buskin A, Zhu L, Chichagova V, Basu B, Mozaffari-Jovin S, Dolan D, et al. Disrupted alternative splicing for genes implicated in splicing and ciliogenesis causes PRPF31 retinitis pigmentosa. Nat Commun. 2018;9:4234

6. Lopez-Mejia IC, Vautrot V, De Toledo M, Behm-Ansmant I, Bourgeois CF, Navarro $\mathrm{CL}$, et al. A conserved splicing mechanism of the LMNA gene controls premature aging. Hum Mol Genet. 2011;20:4540-55.

7. Liu MM, Zack DJ. Alternative splicing and retinal degeneration. Clin Genet. 2013:84:142-9.

8. Pistoni M, Ghigna C, Gabellini D. Alternative splicing and muscular dystrophy. RNA Biol. 2010;7:441-52.

9. Lehalle D, Wieczorek D, Zechi-Ceide RM, Passos-Bueno MR, Lyonnet S, Amiel J, et al. A review of craniofacial disorders caused by spliceosomal defects. Clin Genet. 2015;88:405-15.

10. Lee S, Han S, Jeong AL, Park JS, Yang Y. Depletion of IK causes mitotic arrest through aberrant regulation of mitotic kinases and phosphatases. FEBS Lett. 2014:588:2844-50.

11. Yeh PC, Yeh CC, Chen YC, Juang YL. RED, a spindle pole-associated protein, is required for kinetochore localization of MAD1, mitotic progression, and activation of the spindle assembly checkpoint. J Biol Chem. 2012;287:1170416.

12. Ulrich AKC, Schulz JF, Kamprad A, Schutze T, Wahl MC. Structural basis for the functional coupling of the alternative splicing factors Smu1 and RED. Structure. 2016;24:762-73.

13. Fournier G, Chiang C, Munier S, Tomoiu A, Demeret C, Vidalain PO, et al. Recruitment of RED-SMU1 complex by influenza A virus RNA polymerase to control viral mRNA splicing. PLoS Pathog. 2014;10:e1004164.

14. Keiper S, Papasaikas P, Will CL, Valcarcel J, Girard C, Luhrmann R. Smu1 and RED are required for activation of spliceosomal $B$ complexes assembled on short introns. Nat Commun. 2019;10:3639.

15. Tajbakhsh S. Skeletal muscle stem cells in developmental versus regenerative myogenesis. J Intern Med. 2009;266:372-89.

16. Ito $\mathrm{Y}$, Kayama T, Asahara H. A systems approach and skeletal myogenesis. Comp Funct Genomics. 2012;2012:759407.

17. Jiwlawat $N$, Lynch E, Jeffrey J, Van Dyke JM, Suzuki M. Current progress and challenges for skeletal muscle differentiation from human pluripotent stem cells using transgene-free approaches. Stem Cells Int. 2018;2018:6241681.

18. Sassoon D, Lyons $G$, Wright WE, Lin V, Lassar A, Weintraub $\mathrm{H}$, et al. Expression of two myogenic regulatory factors myogenin and MyoD1 during mouse embryogenesis. Nature. 1989;341:303-7.

19. Rudnicki MA, Schnegelsberg PN, Stead RH, Braun T, Arnold HH, Jaenisch $\mathrm{R}$. MyoD or Myf-5 is required for the formation of skeletal muscle. Cell. 1993; 75:1351-9.

20. Kiefer JC, Hauschka SD. Myf-5 is transiently expressed in nonmuscle mesoderm and exhibits dynamic regional changes within the presegmented mesoderm and somites I-IV. Dev Biol. 2001;232:77-90.
21. Hammond CL, Hinits Y, Osborn DP, Minchin JE, Tettamanti G, Hughes SM. Signals and myogenic regulatory factors restrict pax3 and pax7 expression to dermomyotome-like tissue in zebrafish. Dev Biol. 2007;302:504-21.

22. Chal J, Pourquie O. Making muscle: skeletal myogenesis in vivo and in vitro. Development. 2017;144:2104-22.

23. Nabeshima Y, Hanaoka K, Hayasaka M, Esumi E, Li S, Nonaka I, et al. Myogenin gene disruption results in perinatal lethality because of severe muscle defect. Nature. 1993;364:532-5.

24. Katz Y, Wang ET, Silterra J, Schwartz S, Wong B, Thorvaldsdottir H, et al. Quantitative visualization of alternative exon expression from RNA-seq data. Bioinformatics. 2015;31:2400-2.

25. Schiaffino S, Rossi AC, Smerdu V, Leinwand LA, Reggiani C. Developmental myosins: expression patterns and functional significance. Skelet Muscle. 2015;5:22.

26. Will CL, Luhrmann R. Spliceosome structure and function. Cold Spring Harb Perspect Biol. 2011;3(7):a003707. https://doi.org/10.1101/cshperspect.a003707.

27. Wang ET, Sandberg R, Luo S, Khrebtukova I, Zhang L, Mayr C, et al. Alternative isoform regulation in human tissue transcriptomes. Nature. 2008; 456:470-6.

28. Montes M, Sanford BL, Comiskey DF, Chandler DS. RNA splicing and disease: animal models to therapies. Trends Genet. 2019:35:68-87.

29. Chen M, Manley JL. Mechanisms of alternative splicing regulation: insights from molecular and genomics approaches. Nat Rev Mol Cell Biol. 2009;10: $741-54$

30. Hegele A, Kamburov A, Grossmann A, Sourlis C, Wowro S, Weimann M, et al. Dynamic protein-protein interaction wiring of the human spliceosome. Mol Cell. 2012;45:567-80.

31. Bertram $\mathrm{K}$, Agafonov DE, Dybkov $\mathrm{O}$, Haselbach $\mathrm{D}$, Leelaram MN, Will CL, et al. Cryo-EM structure of a pre-catalytic human spliceosome primed for activation. Cell. 2017:170:701-13 e711.

32. Ka HI, Lee S, Han S, Jeong AL, Park JY, Joo HJ, et al. Deubiquitinase USP47stabilized splicing factor IK regulates the splicing of ATM pre-mRNA. Cell Death Discov. 2020;6:34

33. Friedrichs F, Zugck C, Rauch GJ, Ivandic B, Weichenhan D, Muller-Bardorff M, et al. HBEGF, SRA1, and IK: three cosegregating genes as determinants of cardiomyopathy. Genome Res. 2009:19:395-403.

34. Brogna S, Wen J. Nonsense-mediated mRNA decay (NMD) mechanisms. Nat Struct Mol Biol. 2009:16:107-13.

35. Liu H, Luo M, Wen JK. mRNA stability in the nucleus. J Zhejiang Univ Sci B. 2014;15:444-54

36. Klauer AA, van Hoof A. Degradation of mRNAs that lack a stop codon: a decade of nonstop progress. Wiley Interdiscip Rev RNA. 2012;3:649-60.

37. Trapnell C, Williams BA, Pertea G, Mortazavi A, Kwan G, van Baren MJ, et al. Transcript assembly and quantification by RNA-Seq reveals unannotated transcripts and isoform switching during cell differentiation. Nat Biotechnol. 2010;28:511-5.

38. Watanabe T, Kimura A, Kuroyanagi $\mathrm{H}$. Alternative splicing regulator RBM20 and cardiomyopathy. Front Mol Biosci. 2018;5:105.

39. Yang J, Hung LH, Licht T, Kostin S, Looso M, Khrameeva E, et al. RBM24 is a major regulator of muscle-specific alternative splicing. Dev Cell. 2014;31:8799.

40. Lin JC, Tarn WY. RBM4 down-regulates PTB and antagonizes its activity in muscle cell-specific alternative splicing. J Cell Biol. 2011:193:509-20.

41. Spellman R, Smith CW. Novel modes of splicing repression by PTB. Trends Biochem Sci. 2006:31:73-6.

42. Lin S, Fu XD. SR proteins and related factors in alternative splicing. Adv Exp Med Biol. 2007:623:107-22.

43. Zhong XY, Ding JH, Adams JA, Ghosh G, Fu XD. Regulation of SR protein phosphorylation and alternative splicing by modulating kinetic interactions of SRPK1 with molecular chaperones. Genes Dev. 2009;23:482-95.

44. Brauch KM, Karst ML, Herron KJ, de Andrade M, Pellikka PA, Rodeheffer RJ, et al. Mutations in ribonucleic acid binding protein gene cause familial dilated cardiomyopathy. J Am Coll Cardiol. 2009;54:930-41.

45. Li D, Morales A, Gonzalez-Quintana J, Norton N, Siegfried JD, Hofmeyer M, et al. Identification of novel mutations in RBM20 in patients with dilated cardiomyopathy. Clin Transl Sci. 2010:3:90-7.

46. Hernandez-Hernandez JM, Garcia-Gonzalez EG, Brun CE, Rudnicki MA. The myogenic regulatory factors, determinants of muscle development, cell identity and regeneration. Semin Cell Dev Biol. 2017;72:10-8.

47. Buckingham M, Relaix F. PAX3 and PAX7 as upstream regulators of myogenesis. Semin Cell Dev Biol. 2015;44:115-25. 
48. Ziman MR, Fletcher S, Kay PH. Alternate Pax7 transcripts are expressed specifically in skeletal muscle, brain and other organs of adult mice. Int J Biochem Cell Biol. 1997;29:1029-36.

49. Du S, Lawrence EJ, Strzelecki D, Rajput P, Xia SJ, Gottesman DM, et al. Coexpression of alternatively spliced forms of PAX3, PAX7, PAX3-FKHR and PAX7-FKHR with distinct DNA binding and transactivation properties in rhabdomyosarcoma. Int J Cancer. 2005;115:85-92.

50. Mao C, Hu X, Li N. Identification and expression profile of a novel alternative splicing of Pax7 in chick skeletal muscle. Poult Sci. 2008;87:191925.

51. Fernandes JM, Kinghorn JR, Johnston IA. Differential regulation of multiple alternatively spliced transcripts of MyoD. Gene. 2007;391:178-85.

52. Meedel TH, Farmer SC, Lee JJ. The single MyoD family gene of Ciona intestinalis encodes two differentially expressed proteins: implications for the evolution of chordate muscle gene regulation. Development. 1997;124: $1711-21$.

53. Kimmel CB, Ballard WW, Kimmel SR, Ullmann B, Schilling TF. Stages of embryonic development of the zebrafish. Dev Dyn. 1995;203:253-310.

54. Zuris JA, Thompson DB, Shu Y, Guilinger JP, Bessen JL, Hu JH, et al. Cationic lipid-mediated delivery of proteins enables efficient protein-based genome editing in vitro and in vivo. Nat Biotechnol. 2015;33:73-80.

55. Ha JS, Lee JS, Jeong J, Kim H, Byun J, Kim SA, et al. Poly-sgRNA/siRNA ribonucleoprotein nanoparticles for targeted gene disruption. J Control Release. 2017;250:27-35.

56. Pattanayak V, Lin S, Guilinger JP, Ma E, Doudna JA, Liu DR. High-throughput profiling of off-target DNA cleavage reveals RNA-programmed Cas9 nuclease specificity. Nat Biotechnol. 2013;31:839-43.

57. Thorvaldsdottir H, Robinson JT, Mesirov JP. Integrative Genomics Viewer (IGV): high-performance genomics data visualization and exploration. Brief Bioinform. 2013;14:178-92.

58. Katz Y, Wang ET, Airoldi EM, Burge CB. Analysis and design of RNA sequencing experiments for identifying isoform regulation. Nat Methods. 2010;7:1009-15.

59. Thisse C, Thisse B, Schilling TF, Postlethwait JH. Structure of the zebrafish snail1 gene and its expression in wild-type, spadetail and no tail mutant embryos. Development. 1993;119:1203-15.

60. Kemp HA, Carmany-Rampey A, Moens C. Generating chimeric zebrafish embryos by transplantation. J Vis Exp. 2009;(29). https://doi.org/10.3791/13 94.

\section{Publisher's Note}

Springer Nature remains neutral with regard to jurisdictional claims in published maps and institutional affiliations.

Ready to submit your research? Choose BMC and benefit from:

- fast, convenient online submission

- thorough peer review by experienced researchers in your field

- rapid publication on acceptance

- support for research data, including large and complex data types

- gold Open Access which fosters wider collaboration and increased citations

- maximum visibility for your research: over $100 \mathrm{M}$ website views per year

At $\mathrm{BMC}$, research is always in progress.

Learn more biomedcentral.com/submissions 